\title{
Caractérisation alimentaire et nutritive du légume traditionnel lagenaria siceraria à Brazzaville (Congo)
}

\author{
Y. S. ITOUA OKOUANGO ${ }^{1,2,3^{*}}$, Vital MANANGA ${ }^{1,2,3}$, Michel ELENGA $^{1,2,3}$ et \\ Leila Samira ADIALO ${ }^{2,3}$
}

${ }^{I}$ Équipe Pluridisciplinaire de Recherche en Alimentation et Nutrition (Eprancongo)

${ }^{2}$ Laboratoire de Nutrition et d'Alimentation Humaines BP: 69, Faculté des Sciences et Techniques, Université Marien NGOUABI, Brazzaville, Congo.

3 Département des Masters, Parcours: Sciences Biologiques.

*Auteur correspondant ; E-mail: yvonvasther@gmail.com, Tél :00 (242)06-686-74-37

\section{RESUME}

Au Congo, 166 espèces appartenant à 55 familles de plantes alimentaires sont consommées dans les différentes localités. L'objectif de cette étude est de réaliser une caractérisation alimentaire et nutritive de Lagenaria siceraria. Une enquête de consommation des feuilles de Lagenaria siceraria a été réalisée dans 118 ménages et les analyses physico chimiques ont été effectuées. Ces analyses ont porté sur l'aspect morphologique, les teneurs en eau, en protéines, en glucides, en lipides et en cendres. Les résultats révèlent que ce légume est beaucoup consommé par les jeunes $(42,37 \%)$ dont l'âge est inférieur ou égal à 30 ans. Le niveau d'instruction des enquêtés le plus représenté est celui du lycée avec un pourcentage de 37,3\%. La majorité des personnes enquêtées exerce une activité informelle 48,3\%. Lagenaria siceraria est connu par 93,2\% des personnes enquêtées et 59,3\% des enquêtés consomment ce légume. Les feuilles de Lagenaria siceraria contiennent des protéines (24,5 g/100 g de MS), des glucides (63,22 g/100 g de MS), des cendres $(10,5 \pm 0,15 \mathrm{~g} / 100 \mathrm{~g}$ de MS). Les feuilles de Lagenaria siceraria ont une bonne valeur nutritive, grâce à leur richesse en protéines et en minéraux et pourront contribuer à l'amélioration de l'état nutritionnel des populations.

(c) 2019 International Formulae Group. All rights reserved

Mots clés : légume traditionnel, enquête alimentaire, consommation, valeur nutritive.

\section{Food and nutritional characterization of Lagenaria of siceraria in Brazzaville (Congo)}

\begin{abstract}
In Congo, 166 species belonging to 55 families of food plants are consumed in different localities. The objective of this study is to carry out a food and nutritional characterization of Lagenaria siceraria. A consumption survey of leaves of Lagenaria siceraria was carried out in 118 households and physico-chemical analyzes were carried out, too. These analyzes focused on the morphological aspect and on water, proteins, carbohydrates, lipids and ashes contents. The results reveal that this vegetable is much consumed by young people $(42.37 \%)$ whose age is less than or equal to 30 years. Most of the represented respondents attended
\end{abstract}


senior high school (37.3\%). The majority of the people surveyed have an informal activity (48.3\%). Lagenaria siceraria is known by $93.2 \%$ of those surveyed and $59.3 \%$ of respondents consume this vegetable. The leaves of Lagenaria siceraria contain proteins (24.5 g / $100 \mathrm{~g} \mathrm{DM})$, carbohydrates (63.22 g / $100 \mathrm{~g} \mathrm{DM}$ ), and ashes $(10.5 \pm 0.15 \mathrm{~g} / 100 \mathrm{~g} \mathrm{DM})$. The leaves of Lagenaria siceraria have a good nutritional value, thanks to their proteins and minerals contents and can contribute to the improvement of the nutritional state of the populations. (C) 2019 International Formulae Group. All rights reserved

Keywords: traditional vegetable, food survey, consumption, nutritional value.

\section{INTRODUCTION}

Les légumes feuilles font partie de l'alimentation de nombreuses familles africaines. Ils représentent une part importante à la fois quantitative et qualitative des aliments consommés par les populations. Les légumes feuilles comprennent environ 1025 espèces cultivées ou sauvages en Afrique dont 275 espèces proviennent de l'Afrique tropicale. Parmi ces dernières, 207 sont consommées pour leurs feuilles et 68 sont utilisés à d'autres fins (Mahyao et al., 2009 ; Prota, 2004 ; Kahane et al., 2005).

La qualité des produits alimentaires est devenue de nos jours une préoccupation pour les consommateurs. Les produits de la filière agro-industrielle, bon marché, sont de plus en plus consommés surtout dans les pays en voie de développement. Ce changement d'habitude alimentaire a des répercutions socioéconomiques très importantes sur la population » (Horman, 2004). De nos jours, l'attention et les moyens sont concentrés sur un nombre limité d'espèces d'intérêt commercial, parmi lesquelles on retrouve les légumes occidentaux: le choux, le poireau et la laitue dominent, tandis que l'oseille, l'amarante, l'épinard rejoint le clan des recettes régionales (Baskar, 2005). Or, ces légumes feuilles traditionnelles constituent en général une source de micronutriments (FAO/OMS, 2007). Cette évolution entraîne des risques à la fois de dépendance économique, de malnutrition, de perte d'identité sociale mais aussi d'appauvrissement génétique (Maundu et al., 2004).

Au Congo Brazzaville, on compte 166 espèces appartenant à 55 familles de plantes alimentaires consommées dans les différentes localités (Nkeoua et Boundzanga, 2000).

L'étude de la valeur alimentaire d'un légume traditionnel comme Lagenaria siceraria permet d'avoir des résultats susceptibles d'entrer dans la base de données alimentaires. Or, au Congo, aucune étude sur la valeur alimentaire de ce légume traditionnel n'a été réalisée.

La présente étude a été initiée avec l'ambition de donner un aperçu analytique sur la valeur nutritive du légume traditionnel Lagenaria siceraria consommé au Congo Brazzaville ainsi que sa place dans les habitudes alimentaires des populations de Brazzaville.

\section{MATERIEL ET METHOCDES \\ Présentation du milieu d'étude}

L'étude a été réalisée auprès des habitants de Brazzaville dans deux arrondissements situés au Sud et au Nord de la ville (Bacongo et Talangaï). Brazzaville est la capitale politique et administrative de la République du Congo. Elle se situe dans le Sud du Congo, sur les rives du fleuve Congo, en face de Kinshasa, la capitale de la République Démocratique du Congo. Sa superficie est de $269,3 \mathrm{Km}^{2}$ et compte 1.696.392 habitants (2015) avec une densité de $26428 \mathrm{hab} / \mathrm{Km}^{2}$. (Frey, 1980).

\section{Bacongo}

L'arrondissement Bacongo est l'un des tout premiers avec Poto-Poto. Il héberge le plus grand marché de la ville: le Marché Total. Il comporte le vieux Bacongo (quartier Dahomey) et des quartiers plus récents (Bacongo-moderne ou Mpissa). L'arrondissement Bacongo a une superficie de $7,39 \mathrm{~km}^{2}$ pour une population de 80000 habitants. Il est limité au Nord par 
l'arrondissement 3 Poto-Poto, au Sud et à l'Est par le fleuve Congo et à l'Ouest par l'arrondissement Makélékélé. Au plan administratif, il est subdivisé en 9 quartiers, 63 zones et 475 blocs.

\section{Talangaï}

Le sixième arrondissement Talangaï est situé au Nord de la ville. Par l'importance de sa population, il vient au deuxième rang après Makélékélé. L'arrondissement Talangaï est limité au Nord par l'arrondissement Djiri, au Sud par l'arrondissement Ouenzé, à l'Est par le fleuve Congo et à l'Ouest par le pont de la rivière Mikalou. Il a une population de 500.000 habitants. Talangaï est subdivisé en 8 quartiers.

\section{Matériels \\ Matériel didactique}

Le matériel didactique a été constitué d'une fiche d'enquête, composée des questions ouvertes et des questions fermées qui sont posées à l'enquêté et dont les réponses sont notées ou cochées sur la fiche par l'enquêteur. Les grands points de cette fiche ont été : connaissance du légume, utilité de la plante, ses modes de préparation en tenant compte de ses caractères organoleptiques et de ses fréquences de consommation.

\section{Matériel biologique}

Il a été constitué des feuilles de Lagenaria siceraria. Ces feuilles (photographie1) ont été achetées au marché Total (Bacongo) et transportées au laboratoire pour les analyses physicochimiques.

\section{Méthodologie \\ Enquête dans les ménages}

Le travail sur le terrain a été caractérisé par une enquête de consommation des feuilles de Lagenaria siceraria appelé en langue vernaculaire Ntiya ou Ngapara. L'enquête s'est déroulée du 10 décembre au 15 décembre 2017 auprès de 118 personnes. L'enquête a été réalisée dans les ménages à Bacongo et à Talangaï. L'enquête consiste à rencontrer l'enquêté chez lui. Dans chaque ménage, un seul consommateur était interrogé, désigné par la personne qui s'occupe habituellement des repas. L'enquêté est soumis aux questionnaires qui suivent la chronologie de la fiche d'enquête et les réponses sont notées par l'enquêteur. Le dialogue s'est déroulé en langue officielle (Français) ou en langue nationale (Lingala et Kituba) suivant la langue maîtrisée par l'enquêté.

\section{Type d'enquête}

Nous avons utilisé une enquête transversale. Ce type d'enquête a été choisi par ce qu'elle a été effectuée sur une partie de la population, pendant une durée de cinq jours.

\section{Choix de la zone d'étude et population cible}

Les deux communes ont été choisies par rapport à la localisation de deux importants marchés qui approvisionnent la ville de Brazzaville notamment le grand marché de Total à Bacongo et le grand marché de Texaco à Talangaï. L'enquête a concerné 118 ménages tout sexe et âge confondus.

\section{Critères d'inclusion}

Les personnes soumises à l'enquête doivent répondre aux critères suivants :

- Résider dans les arrondissements Bacongo ou Talangaï au moment de l'enquête.

- Accepter de répondre aux questionnaires.

\section{Lieu d'identification}

L'échantillon végétal a été acheté au marché de total, amené au laboratoire de Botanique du Centre d'Etude sur les Ressources Végétales (CERVE) de la Délégation Générale de la Recherche Scientifique et Technique à Brazzaville en vue de la détermination de l'espèce et des caractéristiques physiques.

Préparation des échantillons pour les analyses chimiques

Les feuilles de Lagenaria siceraria ont été pesées puis séchées à l'étuve à la température de $70{ }^{\circ} \mathrm{C}$ jusqu'à la stabilisation de la masse. A l'issu de ce séchage, les feuilles ont été broyées. La poudre obtenue a été utilisée pour les analyses chimiques.

Détermination de la teneur en eau

La teneur en eau a été déterminée par un séchage des feuilles de Lagenaria siceraria à l'étuve à la température de $70{ }^{\circ} \mathrm{C}$. 
Détermination de la teneur en protéines

La teneur en protéines a été déterminée par la méthode de KJELDAHL (AOAC, 1990).

\section{Détermination de la teneur en lipides}

La méthode de Soxhelet a été utilisée pour déterminer la teneur en lipides des feuilles de Lagenaria siceraria.

Détermination de la teneur en sels minéraux totaux (cendres)

Le taux de cendres est un résidu obtenu après calcination des feuilles de Lagenaria siceraria au four à $500{ }^{\circ} \mathrm{C}$

Détermination de la teneur en glucides totaux

Les sucres ont été extraits par leur solubilité dans l'éthanol après delipidation du broyat des feuilles de lagenaria siceraria

Détermination de la valeur énergétique

La valeur énergétique correspondante a été calculée en utilisant le coefficient de Merrill et Watt (1955) pour les protéines, les lipides et des glucides.

\section{Analyse statistique}

Le dépouillement s'est effectué avec la création d'une base de données dans le logiciel Epi-info 6. Le test de Student ou de comparaison a été utilisé. Il permet de décider si la différence observée entre les deux mouvements est attribuable à une cause systématique ou si elle peut être considérée comme l'effet d'une fluctuation due au hasard.

\section{RESULTATS}

Caractéristiques sociodémographiques des consommateurs des feuilles de Lagenaria siceraria

Les résultats des caractéristiques démographiques des consommateurs des feuilles de Lagenaria siceraria sont présentés dans le Tableau 1: Ces résultats révèlent que, pour l'effectif interviewé (118 personnes enquêtées), la consommation des feuilles de Lagenaria siceraria au cours de l'enquête s'observait à $42,4 \%$ chez les jeunes dont l'âge est inférieur ou égale à 30 ans et à $25,4 \%$, chez les adultes d'un âge compris entre 30 et 40 ans. Les $32,2 \%$ restants ont été d'un âge supérieur à 40 ans, avec une différence très significative, $\mathrm{P}<0,001$.

S'agissant du sexe, Il ressort que sur 118 personnes enquêtées, il y a plus de personnes du genre féminin $(74,6 \%)$ que de personnes du genre masculin $(29,3 \%)$.

De façon générale, $90,7 \%$ des consommateurs étaient scolarisés et $9,3 \%$ non scolarisés. En effet, $6,8 \%$ d'entre eux ont un niveau d'étude primaire, $23,7 \%$ ont un niveau d'étude secondaire premier cycle, $37,3 \%$ ont un niveau d'étude secondaire second cycle et $22,9 \%$ ont pu faire un cycle universitaire. Les personnes qui n'ont jamais été à l'école représentent 9,3\%. La plupart des consommateurs de notre étude sont des célibataires $(41,5 \%)$, suivi des unions libres $(25,4 \%)$ et des mariés (22\%). Les veufs (ves) et les divorcés (es) sont les moins représentés $(7,6 \%$ et $3,4 \%$ respectivement), dont la différence est hautement significative, $\mathrm{P}<0,001$.

Près de la moitié des consommateurs enquêtés exercent une activité informelle $(48,3 \%)$ tandis que $20,3 \%$ sont des commerçants, $16,1 \%$ sont des fonctionnaires et $15,3 \%$ travaillent dans les secteurs privés. Les consommateurs de lagenaria siceraria vivent dans des ménages constitués en moyenne de $4,5 \pm 2,75$ membres.

\section{Pratique alimentaire du légume-feuille} lagenaria siceraria

Connaissance, consommation, mode de consommation et goût du légume-feuille lagenaria siceraria

Le Tableau 2 montre les résultats de la connaissance, la consommation, le mode de consommation et le goût du légume traditionnel Lagenaria siceraria. De ces résultats, la majorité des consommateurs enquêtés $(93,2 \%)$ connaissent ce légume contre $6,8 \%$ qui ne le connaissent pas. Plus de la moitié des enquêtés $(59,3 \%)$ consomment les feuilles de Lagenaria siceraria comme légume, contre $40,7 \%$ qui n'en consomment pas. Généralement les feuilles de Lagenaria siceraria sont consommées cuites par tous les ménages qui en consomment (100\%). 
La majorité des ménages enquêtés $(88,6 \%)$ affirme que ce légume à un goût sucré. Par contre, $17,8 \%$ des consommateurs enquêtés affirment qu'il n'a pas un goût fondamental spécifique (goût insipide), avec $\mathrm{P}<0,001$.

Types d'aliments associés à la préparation et d'accompagnement du légume lagenaria siceraria

Les différents types d'aliments associés à la préparation et d'accompagnement du légume lagenaria siceraria sont présentés dans le Tableau 3. Il y ressort que lors de la préparation du légume Lagenaria siceraria, $35,7 \%$ de ménages enquêtés n'associent pas un autre aliment à la préparation de ce légume-feuille. 30\% l'associent avec de la viande, $18,6 \%$ mettent le poisson frais, $12,9 \%$ le préparent avec le poisson fumé et seulement $2,9 \%$ en consomment par association avec le poisson salé, le test de student révèle une différence hautement significative.

La moitié des ménages (50\%) accompagnent ce plat avec le foufou (farine de manioc préparée). 38,6\% accompagnent le plat avec le manioc, $10 \%$ préfèrent le pain et $1,4 \%$ l'accompagnent avec un autre aliment de base comme le taro, l'igname, la banane plantain.

\section{Fréquence hebdomadaire, Période de consommation et autre utilité des feuilles de lagenaria siceraria}

Le Tableau 4 montre les résultats de la fréquence, période de consommation et autre utilité des feuilles de lagenaria siceraria. Ces résultats montrent que les ménages qui ont consommé le légume étudié une fois dans la semaine sont les plus représentés $(58,6 \%)$, suivis des ménages qui l'ont consommé deux fois dans la semaine $(27,1 \%)$. Les ménages qui ont consommé le légume étudié trois fois et plusieurs fois dans la semaine sont faiblement représentés avec un pourcentage respectif de $12,9 \%$ et $1,4 \%$.

La plupart des ménages enquêtés (70\%) ne consomment pas ce légume pendant toutes les saisons, cela est dû à sa rareté pendant la saison sèche, ce qui a pour conséquence le coût élevé d'achat sur le marché. et $30 \%$ des ménages le consomment pendant toutes les saisons de l'année, $(\mathrm{P}<0,001)$.

Ce légume traditionnel, fait partie de la flore spontanée, pousse sans aucun soin humain, est abondant pendant la saison des pluies. De ce fait, il est généralement beaucoup consommé pendant la saison des pluies $(95,9 \%$ ) contre $4,1 \%$ le consomment pendant la saison sèche.

En dehors de l'utilité alimentaire qui représente $95,7 \%$, un petit nombre de la population enquêté $(4,3 \%)$ l'utilise pour des vertus médicinales. Ces personnes affirment que la consommation du légume étudié soigne la constipation.

\section{Aspects morphologiques des feuilles de lagenaria siceraria}

Le Tableau 5 montre des dimensions des feuilles lagenaria siceraria. Les résultats de ce tableau montrent que ces feuilles présentent des caractéristiques suivantes : longueur $13,9 \pm 2,45 \quad \mathrm{~cm}, \quad$ largeur $12,47 \pm 1,86 \mathrm{~cm}$, longueur du pétiole: $10,9 \pm 1,8$ $\mathrm{cm}$ et le nombre de nervures $3 \pm 0,00$.

Composition nutritionnelle globale et valeur énergétique des feuilles de lagenaria siceraria

Le Tableau 6 présente la composition nutritionnelle globale et la valeur énergétique des feuilles de Lagenaria siceraria. Il ressort de l'analyse de ce tableau que les feuilles de Lagenaria siceraria ont une teneur en eau de $87,07 \mathrm{~g} / 100 \mathrm{~g}$ de matière fraîche. Cependant, les protéines, les glucides et les lipides montrent des teneurs respectives de $24,5 \mathrm{~g} / 100 \mathrm{~g}$ de $\mathrm{MS} ; 63,22 \mathrm{~g} / 100 \mathrm{~g}$ de MS et $1,93 \mathrm{~g} / 100 \mathrm{~g}$ de MS. Les cendres des feuilles de Lagenaria siceraria ont une teneur de $10,35 \mathrm{~g} / 100 \mathrm{~g}$ de MS. Ces nutriments (lipides, protéines, glucides) apportent $364,25 \mathrm{Kcal}$ (soit $1539,26 \mathrm{Kj}$ ). 
Tableau 1: Caractéristiques sociodémographiques des consommateurs du légume traditionnel lagenaria siceraria.

\begin{tabular}{|c|c|c|c|c|}
\hline Variables & & Effectif & Pourcentage(\%) & Significativité \\
\hline \multirow[t]{3}{*}{ Age } & $\leq 30$ ans & 50 & 42,4 & \\
\hline & $<3 \mathrm{O} \leq 40$ ans & 30 & 25,4 & $\mathrm{P}<0,001$ \\
\hline & $>40$ ans & 14 & 32,2 & \\
\hline \multirow[t]{2}{*}{ Sexe } & Masculin & 30 & 25,4 & \\
\hline & Féminin & 88 & 74,6 & $\mathrm{P}<0001$ \\
\hline Niveau & Primaire & 8 & 6,8 & \\
\hline \multirow[t]{4}{*}{ d'instruction } & Collège & 28 & 23,7 & \\
\hline & Lycée & 44 & 37,3 & \\
\hline & Supérieur & 27 & 22,9 & \\
\hline & Jamais scolarisé & 11 & 9,3 & $\mathrm{P}<0001$ \\
\hline Situation & Célibataire & 49 & 41,5 & \\
\hline \multirow[t]{4}{*}{ matrimoniale } & Divorcé & 4 & 3,4 & \\
\hline & Union libre & 30 & 25,4 & $\mathrm{P}<0001$ \\
\hline & Marié & 26 & 22,4 & \\
\hline & Veuf (ve) & 09 & 7,6 & \\
\hline Activité & Fonctionnaire & 19 & 16,1 & \\
\hline \multirow[t]{3}{*}{ professionnelle } & Secteur privé & 18 & 15,3 & \\
\hline & Informel & 57 & 48,3 & $P<0001$ \\
\hline & Commerçant & 24 & 20,3 & \\
\hline Effectif & Moyen & \multicolumn{3}{|c|}{ Dans le Ménage $=4,5 \pm 2,78$ Personnes } \\
\hline
\end{tabular}

Tableau 2: Connaissance, consommation, mode de consommation et goût du légume-feuille lagenaria siceraria.

\begin{tabular}{lcll}
\hline Variables & \multicolumn{1}{c}{ Effectif } & Pourcentage(\%) & significativité \\
\hline \multicolumn{2}{l}{ Connaissance du légume } & & \\
oui & 110 & 93,2 & $\mathrm{P}<0001$ \\
non & 08 & 6,8 & \\
Consommation du légume & & \\
oui & 70 & 59,3 & $\mathrm{P}<0001$ \\
non & 48 & 40,7 & \\
Forme de consommation & & \\
cuit & 70 & 100 & \\
crus & 0 & 0 &
\end{tabular}




\section{Goût du légume}

$\begin{array}{llll}\text { Amer } & 0 & 0 & \\ \text { Aigre } & 0 & 0 & \mathrm{P}<0001 \\ \text { Sucré } & 62 & 88,6 & \\ \text { Salé } & 0 & 0 & \\ \text { insipide } & 08 & 11,4 & \end{array}$

Tableau 3 : Types d'aliments associés à la préparation et d'accompagnement du légume lagenaria siceraria.

\begin{tabular}{llll}
\hline Variables & Effectifs & pourcentage(\%) & Significativité \\
\hline Aliments associé à la préparation & & & \\
Aucun aliment associé & 25 & 35,7 & \\
Poisson frais & 13 & 18,6 & $\mathrm{P}<0001$ \\
Poisson fumé & 09 & 12,9 & \\
Viande de bœuf & 21 & 30 & \\
Poisson salé & 02 & 2,8 & \\
Aliment d'accompagnement & & & $\mathrm{P}<0001$ \\
Manioc & 27 & 38,6 & \\
Farine de manioc & 35 & 50,0 & \\
Pain & 7 & 10 & \\
Riz & 00 & 0,0 & \\
Autres (igname, taro) & 1 & 1,4 & \\
\hline
\end{tabular}

Tableau 4 : Fréquence de consommation hebdomadaire, Période de consommation et autre utilité des feuilles de lagenaria siceraria.

\begin{tabular}{lcll}
\hline Variables & Effectifs & Pourcentage(\%) & Significativité \\
\hline Consommation hebdomadaire & 41 & 58,6 & \\
Une fois & 19 & 27,1 & \\
Deux fois & 09 & 12,9 & $\mathrm{P}<0001$ \\
Trois fois & 01 & 1,4 & \\
Plusieurs fois & 00 & 0,0 & \\
Aucune fois & & & \\
Période de consommation & & & \\
Toutes les saisons & 21 & 30 & $\mathrm{P}<0001$ \\
Oui & 49 & 70 & $\mathrm{P}<0001$ \\
Non & & & \\
Type de saison & 03 & 4,1 & - \\
Saison sèche & 67 & 95,9 & \\
Saison des pluies & & & \\
Autres utilités du légume & & 4,2 & \\
Vertus médicinales & 03 & 0,0 & \\
Autres & 00 & & \\
\end{tabular}


Tableau 5 : Aspects morphologiques des feuilles de lagenaria siceraria.

\begin{tabular}{ll}
\hline Caractéristiques & Valeurs \\
\hline Longueur $(\mathrm{cm})$ & $13,9 \pm 2,45$ \\
Largeur $(\mathrm{cm})$ & $12,47 \pm 1,86$ \\
Pétiole $(\mathrm{cm})$ & $10,9 \pm 1,8$ \\
Nombre de nervures & $3 \pm 0,00$ \\
\hline
\end{tabular}

Tableau 6: Composition nutritionnelle globale et valeur énergétique des feuilles de lagenaria siceraria.

\begin{tabular}{ll}
\hline Composition globale & Valeurs \\
\hline Humidité $(\mathrm{g} / 100 \mathrm{~g}$ de MF) & $87,07 \pm 0,52$ \\
Lipides (g/100g de MS) & $1,93 \pm 0,15$ \\
Protéines (g/100g de MS) & $24,5 \pm 0,00$ \\
Glucides ( $/ 100 \mathrm{~g}$ de MS) & $63,22 \pm 0,00$ \\
Cendres (g/100g de MS) & $10,35 \pm 0,55$ \\
Energie $(\mathrm{kcal})$ & 364,25 \\
\hline
\end{tabular}

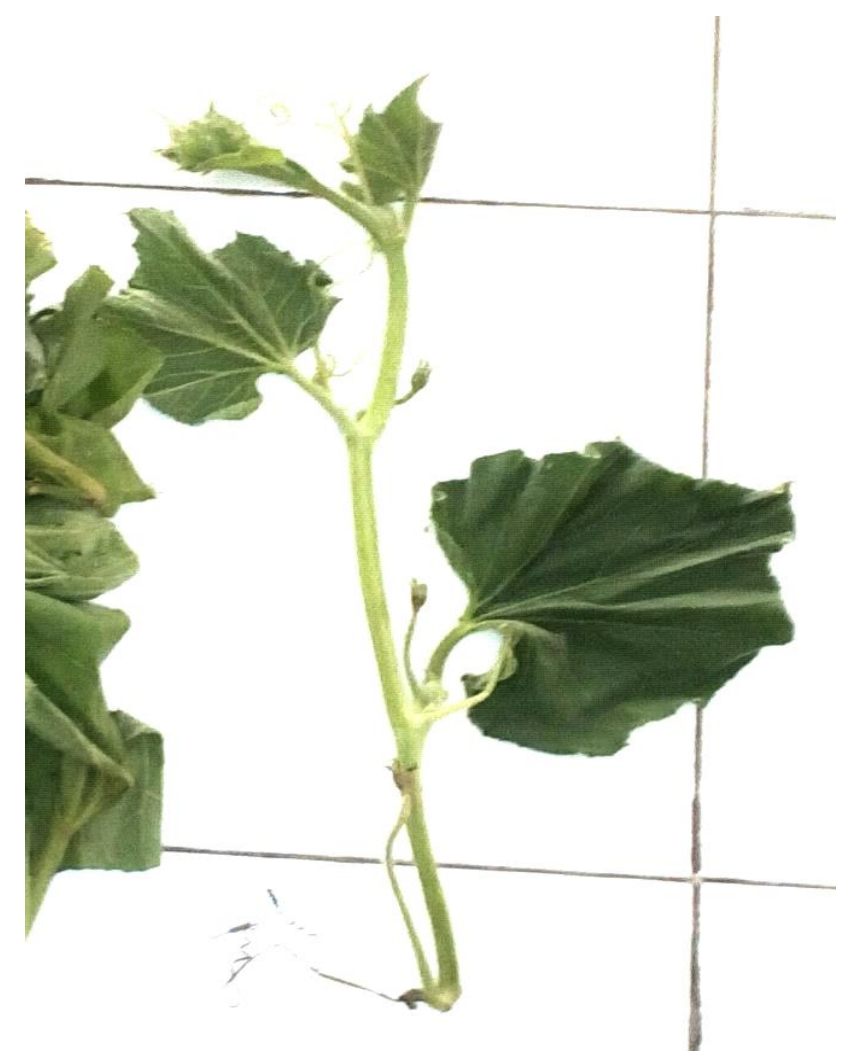

Figure1 : Pieds de Lagenaria siceraria. 


\section{DISCUSSION}

Nos résultats montrent que la tranche d'âge des personnes enquêtées la plus représentée est celle dont l'âge est inférieur ou égale à 30 ans $(42,37 \%)$. Les résultats obtenus sont supérieurs à ceux obtenus par Elenga et al. (2016) sur une étude de Salacia pynaertii. Les personnes enquêtées de sexe féminin sont les plus représentatives $(74,6 \%)$ dans notre étude. Les discussions ont été orientées beaucoup plus vers les femmes parce que ce sont elles qui s'occupent de la cuisine, ce qui rejoint les résultats de Probst (2008) qui disaient que les femmes sont responsabilisées dans le choix des légumes et la cuisson des repas. Les activités liées à l'alimentation sont presqu'exclusivement réservées aux femmes.

Parmi les consommateurs enquêtés, $37,3 \%$ ont un niveau d'étude secondaire second cycle et $22,9 \%$ ont pu faire un cycle universitaire. La plupart des consommateurs de notre étude sont des célibataires $(41,5 \%)$ et près de la moitié des consommateurs enquêtés exercent une activité informelle $(48,3 \%)$. La consommation du légume feuille étudié n'est pas liée au niveau social du foyer ni au niveau d'instruction du sujet. Ainsi nous acceptons l'affirmation selon laquelle en «Afrique les populations ont souvent recours aux espèces végétales pour couvrir leurs besoins alimentaires et assurer la sécurité alimentaire» soutenue par Van Rendsburg et al. (2004).

Lagenaria siceraria est connue par 93,2\% des personnes enquêtées, ce qui signifie que notre plante d'étude n'est pas méconnue par la population d'étude. Ainsi nous pouvons dire que presque la totalité des personnes enquêtées a des connaissances sur le légume traditionnel. Nos résultats concordent avec ceux des travaux de Tchiegang et Kitikil (2004) qui stipulent que lors d'une étude sur les données ethno nutritionnelles et caractéristiques physicochimiques des légumes traditionnels consommés dans la savane de l'adamaoua, $100 \%$ des personnes enquêtées connaissent les légumes traditionnels.

La consommation des feuilles de Lagenaria siceraria est de $59.3 \%$. Le résultat obtenu est inferieur a la consommation des feuilles de Phytolacca dodecandra (86\%) et celui de Salacia pynaertii (100\%), étude réalisée respectivement par Itoua Okouango et al. (2015) et Elenga et al. (2016).

Lagenaria siceraria fait partie des légumes traditionnels couramment consommés à Brazzaville. Ces résultats rejoignent ceux de Mushagalusa Balasha et al. (2015) qui affirment qu'au Congo démocratique, les feuilles de Lagenaria siceraria occupent le deuxième rang parmi les légumes traditionnels les plus consommés.

La forme de consommation du légume étudié est celle du légume cuit (100\%) comparable à celle d'Amaranthus hybridus (100\%), étude faite par Soro et al. (2012) sur évaluation de la composition nutritionnelle des légumes traditionnels. La préparation de Lagenaria siceraria est soit simple, soit garnie de viande, de poisson frais, fûmé, ou salé. L'utilisation de la viande ou du poisson pourrait être influencée par le facteur financier comme l'avait souligné les auteurs Itoua Okouango et al. (2015). L'accompagnement du plat de Lagenaria siceraria se fait avec la Chikwangue ou le foufou (farine de manioc), comme coutume alimentaire des congolais observé par Itoua Okouango et al. (2015) lors d'une étude sur Phytolacca dodacandra.

En ce qui concerne la fréquence de consommation de Lagenaria siceraria, 58,6\% des enquêtés le consomment une fois dans la semaine. Cette frequence est élevée par apport à celle obtenue par Mushagalusa Balasha et al. (2015) (40\%), lors d'une étude sur la consommation alimentaire à Mimbulu. La fréquence de consommation des feuilles de Lagenaria siceraria de notre étude est plus élevée pendant la saison pluvieuse $(95,9 \%)$. Cette densité de la consommation pendant la saison des pluies pourrait s'expliquer par la disponibilité de ce légume sur le marché et son prix d'achat favorable à la population pendant cette période.

En dehors des qualités alimentaires, les résultats montrent que les feuilles de Lagenaria siceraria possèdent des propriétés médicinales. En effet, la consommation des feuilles de Lagenaria siceraria règle le problème de la constipation. Nos résultats 
coïncident avec les affirmations de Soro et al. (2012) et Mensah et al. (2008) selon lesquelles, la consommation des fruits et de légumes peut aider à prévenir plusieurs maladies non transmissibles liées à l'alimentation. Les légumes constituent une source des fibres alimentaires, intervenant au niveau du tractus digestif pour prévenir l'absorption d'un excès de cholestérol.

Concernant les aspects morphologiques et la composition chimique, les feuilles de Lagenaria siceraria sont plus longues (longueur 13,9 $\pm 2,45 \mathrm{~cm}$ ) et larges $(12,47 \pm 1,86 \mathrm{~cm})$ que celles obtenues par Mbemba et al. (2013) sur une étude de Cuervea isangiensis. La teneur en eau est de $87.07 \mathrm{~g} / 100 \mathrm{~g}$ de matière fraiche. Ces résultats sont proches de ceux obtenus par Tchegang et Kitikil (2004) pour Hibiscus S. (87, 63\%). Cependant, Itoua okouango et al. (2015) ont trouvé une valeur de $83,87 \%$ pour Phytolacca dodecandra, Depesay (2007) a indiqué des valeurs qui varient entre 85 et $95 \%$ pour les légumes feuilles. La valeur de notre étude est comprise dans cet intervalle.

La teneur en protéines des feuilles de Lagenaria siceraria est de 24,5g/100g de MS et en glucides elle est $63,22 \mathrm{~g} / 100 \mathrm{~g}$ de MS. Ces valeurs en protéines et en glucides sont proches de celles de Salacia pynaertii obtenues par Elenga et al. (2016) qui sont respectivement $24,07 \mathrm{~g}$ et $67,54 \mathrm{~g} / 100 \mathrm{~g}$ de MS. La teneur en sels minéraux totaux (cendres) des feuilles de Lagenaria siceraria $10,35 \mathrm{~g} / 100 \mathrm{~g}$ de MS est comparable à celle de Cuervea isangiensis $10,04 \mathrm{~g} / 100 \mathrm{~g}$ de MS signalé par MBemba et al. (2012).

Les résultats de nos analyses sur la composition chimique (protéines et glucides) du légume étudié sont supérieurs à ceux de plusieurs espèces de légumes traditionnels consommées en Afrique (Itoua Okouango et al. 2015) et font de Lagenaria siceraria une véritable source de protéines végétales et de glucides non négligeable.

\section{Conclusion}

La consommation des feuilles de Lagenaria siceraria rentre dans les habitudes alimentaires de la population de Brazzaville.
Cette étude a révélé que Lagenaria siceraria est largement consommé à Brazzaville par des personnes ayant des niveaux sociodémographiques différents.Les feuilles de Lagenaria siceraria ont une bonne valeur nutritive, grâce à leur richesse en protéines et en minéraux et pourront contribuer à l'amélioration de l'état nutritionnel des populations.

\section{REFERENCES}

AOAC (Association of Official Analytical Chemists). 1990. (15th edition), Helrich K (Ed). Arlington, Virginia 22201, USA. Baskar Rajan G. 2005. Leafy Vegetables. Ukaaz Pub.: Hyderabad; 178 p.

Depesay L. 2007. Les légumes dans l'alimentation : leurs effets nutritionnels. Fondation Louis Bonduelle.7p.

Elenga M, Itoua Okouango YS, Loubelo Ongnangué LU, Mananga V. 2016. Evaluation of the consumption and physicochemical characteristics of the leaves of Salacia pynaertii in the food practices of populations of Brazzaville (Congo). African Journal of Food Science, 10(10): 194-202.

FAO/OMS. 2007. Report of the international workshop on the promotion of the fruits and vegetables in the countries French-speaking of sub-Saharan Africa, Yaoundé, Cameroon, 27p.

Frey R. 1980. Livre d'or du Centenaire de Brazzaville. PUBLI-CONGO : Brazzaville ; 354p.

Horman D. 2004. Chicken connection. Le poulet africain étouffé par l'Europe. Agrobusiness, dumping, souveraineté alimentaire. Groupe de Recherche pour une Stratégie Economique Alternative (GRESEA) : Bruxelles ; 140p.

Itoua Okouango YS, Elenga M, Moutsamboté JM, Mananga V, Mbemba F. 2015. Évaluation de la consommation et de la composition nutritionnelle des légumesfeuilles de Phytolacca dodecandra L'Herit consommés par les populations originaires des districts d'Owando et de Makoua. Journal of Animal \& Plant Sciences, 27 : 2071-7024. 
Kahane R, Temple L, Brat P, De Bon H. 2005. Les légumes feuilles des pays tropicaux: diversité, richesse économique et valeur santé dans un contexte très fragile. Colloque Angers ; Les légumes : un patrimoine à transmettre et à valoriser, 7-9. 9p.

Mahyao A, Kouamé C, Agbo E, N'zi JC, Fondio L, Van Damme P. 2009. Socioeconomic importance of urban market supply chains of indigenous leafy vegetable of Côte d'Ivoire. Acta Horticulturae, 806: 489-496.

Maundu PM, Ngugi GW, Kabuye CHS. 2004. Traditional Food Plants in Kenya. Kenya Resource Centre for Indigenous Knowledge. National Museums of Kenya: Nairobi; 270 p.

Mbemba F, Tatola NK, Itoua-Okouango S, Massamba D, Nzikou JM, Silou Th, Moutsambote JM, Mvoula-Tsieri M. 2012. Composition in Mineral Elements of the Traditional Vegetables Leaves of Cuervea isangiensis (de wild.) N.hallé in Congo-Brazzaville. Current Research Journal of Biological Sciences, 4(6): 738-742.

Mbemba F, Moutsambote JM, Nzikou JM, Mvoula-Tsiery M, Itoua Okouango S, Nganga I, Mboungou Z, Silou T. 2013. Physical Factures Nutritional Value of the Traditional Picking Vegetable, cuervea isangiensis (De wild.) N. hallé in Congo- Brazzaville. Advance Journal of Food Science and Technology, 5(1): 72-76.

Mensha JK, Okoli RI, Ohaju-obodo, Eifediyi K. 2008. Phytochemical, nutrition al and medical properties of some leaf vegetables consumed by Edo people of Nigeria. Afrc. J. Biot., 7(14): 2304-2309.

Merrill Al, Watt BK. 1955. Energy value of Food, Basis. United States Department of Agriculture: Washington, DC; 74p.
Mushagalusa Balasha A, Kadiata EM, Mbangu DM, Kotho PL, Mushobekwa ZF, Onakudu L, Madi D, Kabala LK, Lenga NK. 2015. Consommation alimentaire à Mimbulu : description des unités de mesure et des modes alimentaires des ménages ruraux du Katanga. International Journal of Innovation and Scientific Research, 19: 364-376.

Nkeoua G, Boundzanga GC. 2000. Données sur les produits forestiers non ligneux en république du Congo. EC - FAO, 124p.

Probst L. 2008. Consumer Willingness to pay for safe vegetables in peri-urban west Africa. The case of Ghana. BOKU/IITA. Final report, $107 \mathrm{p}$.

PROTA. 2004. Ressources Végétales de l'Afrique Tropicale. Légumes (Vol. 2). Grubben GJH, Denton OA. (Eds). Fondation PROTA / Backhuys Publishers / CTA: Wageningen; 737p.

Soro LC, Atchibri Ocho-Anin A, Kouame C, Abgo Adouko E, Kouadio A, Kouassi K. 2012. Valeur nutritionnelle des légumes-feuilles consommés en Côte d'Ivoire. Int. J. Biol. Chem. Sci., 6(1): 128-135.

DOI : http://dx.doi.org/10.4314/ijbcs.v6i1.12

Tchiégang C, Kitikil A. 2004, Données ethnonutritionnelles et caractéristiques Physico-chimiques des légumes-feuilles consommés dans la savane de l'Adamaoua (Cameroun). Tropicultura, 22(1) : 11-18.

Van Rensburg WJ, Venter SL, Netshibuvhi TR, Venter Heever E, Viaster HJ, de Ronde JA. 2004. Role of indigenous leafy vegetables in combatting hunger and malnutrition. S. Afr. J. Bot., 70(1): 52-59. 